\title{
Sources of variability in computed tomography perfusion: implications for acute stroke management
}

\author{
Benjamin Zussman, B.S., ${ }^{1}$ Pascal Jabbour, M.D., ${ }^{2}$ Kiran Talekar, M.D., ${ }^{3}$ \\ Richard Gorniak, M.D., ${ }^{3}$ and Adam E. Flanders, M.D. ${ }^{3}$ \\ ${ }^{1}$ Jefferson Medical College, ${ }^{2}$ Department of Neurosurgery, and ${ }^{3}$ Department of Radiology, Thomas Jefferson \\ University Hospital, Philadelphia, Pennsylvania
}

\begin{abstract}
Object. Although dynamic, first-pass cerebral CT perfusion is used in the evaluation of acute ischemic stroke, a lack of standardization restricts the value of this imaging modality in clinical decision-making. The purpose of this study was to comprehensively review the reported sources of variability and error in cerebral CT perfusion results.

Methods. A systematic literature review was conducted, 120 articles were reviewed, and 23 published original research articles were included. Sources of variability and error were thematically categorized and presented within the context of the 3 stages of a typical CT perfusion study: data acquisition, postprocessing, and results interpretation.

Results. Seven factors that caused variability were identified and described in detail: 1) contrast media, the iodinated compound injected intravascularly to permit imaging of the cerebral vessels; 2) data acquisition rate, the number of images obtained by CT scan per unit time; 3 ) user inputs, the subjective selections that operators make; 4) observer variation, the failure of operators to repeatedly measure a perfusion parameter with precision; 5) software operational mode, manual, semiautomatic, or automatic; 6) software design, the mathematical algorithms used to perform postprocessing; and 7) value type, absolute versus relative values.

Conclusions. Standardization at all 3 stages of the CT perfusion study cycle is warranted. At present, caution should be exercised when interpreting CT perfusion results as these values may vary considerably depending on a variety of factors. Future research is needed to define the role of CT perfusion in clinical decision-making for acute stroke patients and to determine the clinically acceptable limits of variability in CT perfusion results.
\end{abstract}

(DOI: 10.3171/2011.3.FOCUS1136)

KEY WORDS
standardization $\quad$ computed tomography perfusion $\quad$ brain imaging $\quad$ stroke $\quad$

$\mathrm{T}$ HERE are increasing therapeutic options for patients with acute ischemic stroke, including chemical and mechanical intraarterial thrombolysis. ${ }^{1}$ Brain imaging is used to determine therapy eligibility by discriminating between patients who may benefit from therapy and patients who will not benefit or may even be further injured by therapy.

In the setting of acute stroke, the most practical initial imaging test is CT. Noncontrast CT rapidly and accurately identifies intracranial hemorrhage and helps discriminate nonvascular causes of neurological symptoms. Computed tomography angiography detects intra- and extracranial vessel abnormalities, and may guide treatment decisions for endovascular recanalization therapies. Dynamic CT perfusion generates quantitative measures

Abbreviations used in this paper: ASIST = Acute Stoke Imaging Standardization; $\mathrm{CBF}=$ cerebral blood flow; $\mathrm{MCA}=$ middle cerebral artery; STIR $=$ Stroke Imaging Repository. of cerebral perfusion and therefore has the unique potential to differentiate thresholds of reversible and irreversible ischemia. ${ }^{16}$

In conjunction with $\mathrm{CT}$ and $\mathrm{CT}$ angiography, $\mathrm{CT}$ perfusion has been shown to improve the diagnosis of acute stroke, ${ }^{7}$ but the usefulness of CT perfusion in making acute treatment decisions has not yet been established because CT perfusion imaging information about the degree of reversibility of ischemic injury has been limited by a lack of standardization. ${ }^{6}$ Patient selection for reperfusion therapies is not without significant risk because these drugs can cause life-threatening intracranial hemorrhage, and for CT perfusion results to be applied clinically, it is necessary to know that the data are accurate and reproducible. The purpose of this study was to systematically review the reported sources of variability in cerebral CT perfusion results and discuss the clinical implications for acute stroke management. 


\section{B. Zussman et al.}

\section{Methods}

\section{Data Acquisition}

A systematic literature search was conducted. The following search key words were used in a variety of permutations: "CT," "computed tomography," "perfusion," "blood flow," "cerebral," and "stroke." Search limits included human species, English language, and publication dates from 1970 to 2010. The PubMed database was searched, which returned several hundred articles.

The abstracts of all articles were reviewed to identify reports that focused on cerebral CT perfusion, in which cerebral CT perfusion was defined as CT perfusion used in the context of brain imaging. One hundred twenty reports met this criterion. These reports and any relevant references listed within them were closely reviewed to identify original research articles that reported sources of real or potential variability and error in quantitative cerebral CT perfusion values. Twenty-three published original research articles fit these criteria. These studies were reviewed, and reported sources of real or potential variability and error in quantitative cerebral CT perfusion results were recorded (Table 1). In addition, non-peer-reviewed publications produced by the ASIST-Japan group (http://asist.umin.jp/index-e.htm) and the STIR consortium (https://stir.ninds.nih.gov/html/index.html) were reviewed. Non-peer-reviewed data included in this report are identified as such.

\section{Data Organization}

Sources of variability and error were thematically categorized to facilitate conceptualization.

\section{Results}

\section{Variability in Quantitative CT Perfusion Results}

Contrast Media. Contrast media is the iodinated compound injected intravascularly to permit imaging of the cerebral vessels. Contrast media is injected intravenously during CT scanning. Kloska et al. ${ }^{10}$ compared 2 different contrast media concentrations ( 300 vs $370 \mathrm{mg}$ iodine/ $\mathrm{ml}$ ) and found that they yielded equivalent $\mathrm{CT}$ perfusion source-image tissue enhancement in the caudate nucleus, thalamus, and frontal white matter of the nonischemic hemisphere, but significantly different CT perfusion source-image enhancement in the region of the superior sagittal sinus. Konig et al. ${ }^{12}$ compared 300 versus $400 \mathrm{mg}$ iodine $/ \mathrm{ml}$ concentrations and found no significant difference between mean CT perfusion results derived from nonischemic frontal white matter. They did not assess the ischemic hemisphere. At a national stroke imaging meeting in 2007, participants recommended using 350-370 mg/ $\mathrm{ml}$ concentrations, ${ }^{28}$ but many institutions, including the authors', do not (we use $320 \mathrm{mg} / \mathrm{ml}$ ).

Data Acquisition Rate. The data acquisition rate is the number of images obtained by the CT scanner per unit time. It is proportional to image resolution and radiation dose and therefore must be optimized to balance adequate image quality with radiation safety. The data acquisition rate affects CT perfusion results, but the specific relationship is controversial. For example, Wintermark et al. ${ }^{31}$ found that sampling intervals $>1$ second do not cause significant variability in CT perfusion results, while Kloska et al. ${ }^{11}$ found that they do (for example, $\mathrm{CBF}$ values were increased $[\mathrm{p}=$ 0.044-0.001] with $>1$ second intervals). Different studies recommend different data acquisition rates, ranging from 1 image $/ 0.5 \mathrm{sec}^{8}$ to 1 image/ $3 \mathrm{sec}^{27}$

User Inputs. User inputs are the subjective selections that operators make during CT perfusion acquisition and postprocessing. For example, CT operators select the 2-4 cerebral slices that are scanned. Operators also select important inputs that are used to process image data. For instance, radiologists draw regions of interest on top of raw CT images to label 2 functions that are used to generate perfusion maps. These functions are called the arterial input function and the venous output function. Two studies $^{3,30}$ found that varying placement of the arterial input function did not cause significant variability in CT perfusion results. Arterial input function placement distal to an arterial thrombus, however, undermines perfusion calculation assumptions, and causes significant variability in CT perfusion results. ${ }^{4}$ Two additional studies ${ }^{9,19}$ found that varying placement of the venous output function caused significant variability in CT perfusion results. Although the anterior cerebral artery is commonly selected for the arterial input function and the superior sagittal sinus is commonly selected for the venous output function, there is no standard recommendation for selecting these user inputs.

Observer Variation. Observer variation is the failure of operators to repeatedly measure CT perfusion values with precision. Observer variation may occur whenever user inputs are made. Two types of observer variation have been reported: interoperator (between operators), and intraoperator (reproducibility of 1 operator). Several authors have concluded that observer variation causes a minimal amount of variability in CT perfusion results. ${ }^{3,20,21,29}$ For example, Sanelli et al. ${ }^{21}$ found high inter- and intraoperator correlations $(r=0.87-0.99$ and $\mathrm{r}=$ $0.91-0.99$, respectively) and low inter- and intraoperator coefficients of variability $(2.5 \%-9.5 \%$ and $1.4 \%-6.5 \%$, respectively) in CT perfusion results. Other studies ${ }^{5,23}$ report observer coefficients of variability $>30 \%$, suggesting that observer variation causes a substantial amount of variability in CT perfusion results.

Software Operational Mode. The software operational mode refers to manual, semiautomatic, or automatic data processing. For example, many software applications allow manual, semiautomatic, or automatic selection of input functions (for example, arterial input function or venous output function). Two studies ${ }^{21,24}$ found that automated data processing caused more variability in CT perfusion results than manual mode data processing. In contrast, Soares et al. ${ }^{23}$ found more consistent results.

Software Design. Software design is a broad category that refers to the mathematical algorithms used to process the image data. For example, CT perfusion software applications incorporate image data and operator inputs, 
Variability in computed tomography perfusion

TABLE 1: Categorized list of original research articles included in this study*

\begin{tabular}{|c|c|c|c|}
\hline Error Source & Authors \& Year & No. of Patients & Patient Type \\
\hline \multirow[t]{2}{*}{ contrast media } & Kloska et al., $2007^{10}$ & 90 & suspected AIS \\
\hline & Konig et al., 2007 & 21 & suspected AIS \\
\hline \multirow[t]{4}{*}{ data acquisition rate } & Kloska et al., $2010^{11}$ & 32 & suspected AIS \\
\hline & Wiesmann et al., 2008 & 8 & suspected AIS \\
\hline & Kämena et al., 2007 & 30 & suspected AIS \\
\hline & Wintermark et al., $2004^{31}$ & 60 & AIS \\
\hline \multirow[t]{5}{*}{ user inputs } & Ferreira et al., 2010 & 14 & unilateral proximal MCA occlusion \\
\hline & Wintermark et al., $2008^{30}$ & 55 & suspected AIS \\
\hline & Bisdas et al., 2007 & 18 & suspected AIS \\
\hline & Kealey et al., 2004 & 40 & AIS, CO, TIA, SAH \\
\hline & Sanelli et al., $2004^{19}$ & 3 & AIS \\
\hline \multirow[t]{6}{*}{ observer variation } & Soares et al., 2009 & 30 & suspected AIS \\
\hline & Bisdas et al., 2007 & 18 & suspected AIS \\
\hline & Sanelli et al., $2007^{20}$ & 20 & suspected AIS, vasospasm, chronic cerebral ischemia \\
\hline & Sanelli et al., $2007^{21}$ & 20 & suspected AIS, vasospasm, chronic cerebral ischemia \\
\hline & Wintermark et al., $2005^{29}$ & 46 & suspected AIS \\
\hline & Fiorella et al., 2004 & 20 & not specified \\
\hline \multirow[t]{3}{*}{ software operational mode } & Soares et al., 2009 & 30 & suspected AIS \\
\hline & Sanelli et al., $2007^{21}$ & 20 & suspected AIS, vasospasm, chronic cerebral ischemia \\
\hline & Turk et al., 2007 & 33 & CAS \\
\hline \multirow[t]{7}{*}{ software design } & Ferreira et al., 2010 & 14 & unilateral proximal MCA occlusion \\
\hline & Kudo et al., $2010^{14}$ & 10 & AIS \\
\hline & Kudo et al., $2009^{13}$ & 6 & healthy volunteers \\
\hline & Sasaki et al., 2009 & 20 & suspected AIS \\
\hline & van der Schaaf et al., 2006 & 10 & $\begin{array}{l}\text { suspected aneurysms, suspected sinus thrombosis, SAH, intracerebral } \\
\text { hematoma }\end{array}$ \\
\hline & Kudo et al., $2003^{15}$ & 5 & healthy volunteers \\
\hline & Zussman et al., in press & 11 & suspected AIS \\
\hline value type & Waaijer et al., 2007 & 20 & CAS \\
\hline
\end{tabular}

* AIS = acute ischemic stroke; CAS = carotid artery stenosis; $\mathrm{CO}=$ carotid occlusion; $\mathrm{SAH}=$ subarachnoid hemorrhage; $\mathrm{TIA}=$ transient ischemic attack.

and perform multistep mathematical operations to generate perfusion results. Different algorithms, such as the maximum slope algorithm and the deconvolution family of algorithms, generate significantly different CT perfusion results for identical source data. ${ }^{14}$ Similarly, different software applications using different corrective functions for tracer delay (an effect that occurs when contrast media reaches tissues of interest more slowly than predicted) yield significantly different CT perfusion results., ${ }^{43,14,22}$ Different corrective functions for partial-volume effects (inaccurate measurements of contrast media concentration that occur due to the small size or location of cerebral vessels) cause significantly different CT perfusion results. ${ }^{25}$ Computed tomography perfusion software that uses a corrective technique called vascular pixel elimination was found to more closely correlate with PET results than software that does not incorporate this technique..$^{15}$ As a result, different commercial CT perfusion software postprocessing applications have been shown to produce significantly different CT perfusion results for identical source data. ${ }^{14,32}$ Because there is no industry standard for software design, calibration, or validation, quantitative assessment is not comparable across platforms (Fig. 1).

Value Type. The value type refers to absolute versus relative CT perfusion values. For example, when perfusion values for a given region of tissue are directly reported, they are called absolute values. A given region of tissue can also be compared with the corresponding region of tissue on the contralateral cerebral hemisphere, creating a ratio between the 2 hemispheres. When reported, these ratios are called relative values. Waaijer et al. ${ }^{26}$ compared absolute and relative values and found that relative values caused less variability in CT perfusion results than absolute values. This suggests that absolute values of CT perfusion are less reliable and reproducible than relative values and should not be used as a basis for decision-making.

Non-Peer-Reviewed Factors. Additional factors have been shown to affect raw CT image data including tube current and voltage, scan mode (cine vs intermittent), scan timing, and the tomographic reconstruction process, 


\section{B. Zussman et al.}

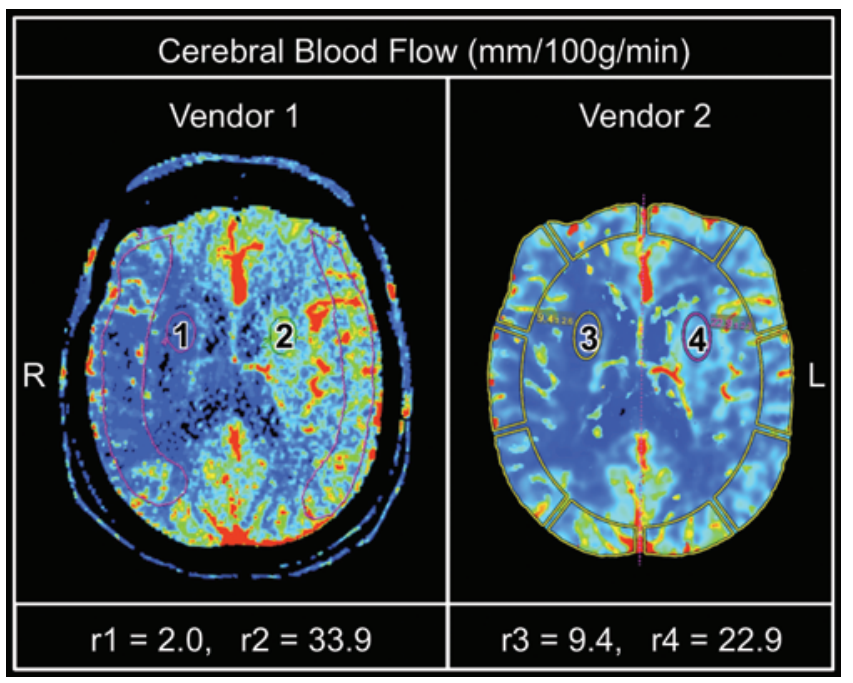

FIG. 1. Different commercial CT perfusion software applications produce significantly different quantitative results for identical source data. The figure shows CBF perfusion color-maps for the same patient data that were reproduced at our institution using 2 different software applications, Vendor 1 (left) and Vendor 2 (right). The maps reveal severe ischemia in the region of the right MCA territory. Regions-of-interest (numbered ovals 1-4) were drawn on the perfusion maps, and software platforms returned perfusion results for the tissue enclosed within each oval. Note that quantitative perfusion results for the ischemic tissue ( $r 1$ and $r 3$ ), as well as for the normal contralateral hemisphere ( $r 2$ and $\mathrm{r} 4$ ) differed considerably by vendor, although the general trend of decreased CBF in the ischemic tissue was consistent for both vendors. There is no industry standard for software design.

all of which affect CT perfusion results (K. Kudo, unpublished data, 2004). Consensus-based expert recommendations for a CT perfusion image acquisition protocol were recently published, ${ }^{28}$ but many institutions have not adopted them.

Additional software design considerations that have been shown to affect $\mathrm{CT}$ perfusion results include the presence and type of preprocessing denoising filter, image matrix size, and deconvolution method (K. Kudo, unpublished data, 2004).

\section{Variability in Qualitative CT Perfusion Results}

In addition to being quantitative, CT perfusion results are also qualitative. For example, perfusion para- metric maps display quantitative CT perfusion values in a color-map overlay that allows qualitative, contextual visualization of an entire brain slice. Computed tomography perfusion color-maps are not standardized and vary by manufacturer, meaning that a given quantitative value will be translated into different colors by different CT perfusion software applications. These parametric color maps are used to qualitatively assess relative variations in CT perfusion metrics. The color scale is not fixed and is operator-adjustable (Fig. 2). Although there is a suggested CT perfusion color scale standard (http://asist.umin.jp/ index-e.htm), it is not in general use and has not been incorporated into commercial software.

\section{Discussion}

Computed tomography perfusion imaging measures cerebral perfusion and calculates quantitative perfusion results. The typical CT perfusion study has 3 stages: data acquisition, postprocessing, and results interpretation. In the data acquisition stage, the patient is scanned and a contrast agent is injected. Image data gathered during this stage is then transferred to a proprietary vendor workstation for postprocessing. In the postprocessing stage, radiologists use software applications to generate quantitative CT perfusion results. These results are then displayed as quantitative values and qualitative, parametric colormaps for results interpretation. In the results interpretation stage clinicians evaluate CT perfusion values and maps and reformat them to their liking, to aid in clinical decision-making. Sources of variability in CT perfusion are introduced at different stages of a CT perfusion study, and Figure 3 shows the sources of variability identified by this study within the context of the 3 CT perfusion stages.

Several recent studies underscore CT perfusion's clinical potential, but also indirectly highlight the great challenges facing CT perfusion. For example, Murphy et al. ${ }^{17}$ studied 30 stroke patients and compared CT perfusion studies (performed at admission) to noncontrast CT studies (performed 5-7 days poststroke). They identified a highly sensitive (95\%) and specific (94\%) CT perfusion threshold for differentiating between ischemic white matter that ultimately infarcted and ischemic white matter that recovered. Aviv et al. ${ }^{2}$ studied 40 stroke patients by comparing admission CT perfusion studies to MR and
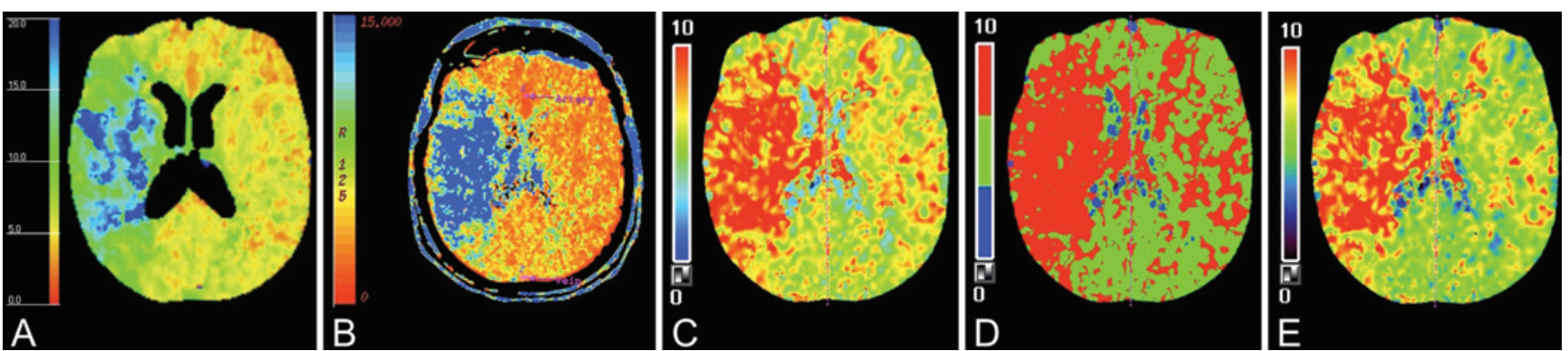

FIG. 2. Different or adjustable color scales mean that identical source data can look very different, depending on the scale chosen. The figure shows mean transit time perfusion color-maps for the same patient data that were reproduced at our institution using different color scale settings. Intervendor color differences are shown: image A was generated using vendor 1 software, image B with vendor 2 software, and images C-E with vendor 3 software. Intravendor color differences are also shown: images $\mathbf{C}-\mathbf{E}$ were generated using 3 different color scales available on the same software platform. 


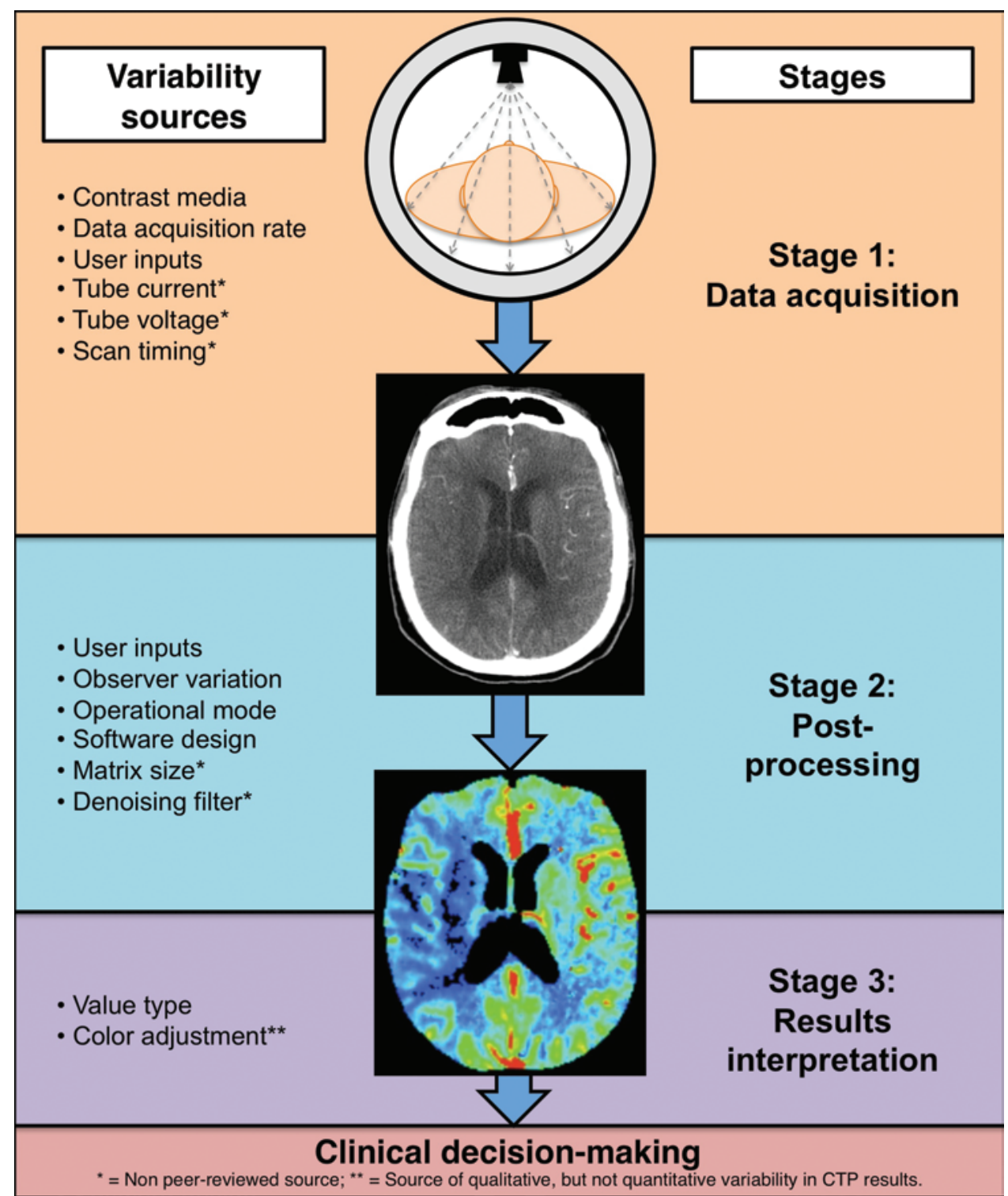

FIG. 3. Illustration of the sources of variability at different stages of a CT perfusion (CTP) study.

CT studies performed at poststroke follow-up. They identified a highly sensitive (77\%) and specific (94\%) CT perfusion threshold for predicting hemorrhagic transformation in acute stroke patients. These and other studies underscore CT perfusion's great potential because they offer a glimpse of how quantitative cerebral perfusion data might influence decision-making in the context of cerebrovascular disease.

While these studies underscore the potential clinical value of CT perfusion, they also highlight a major limitation of this technique, namely variability and error caused by a lack of standardization. For example, the data of Murphy et al. ${ }^{17}$ were analyzed using a single vendor's postprocessing platform, which raises the question of whether the authors would have identified the same results and reached the identical conclusion had a different vendor been chosen for this study. This question may be especially relevant in stroke centers that use multiple ven- dor acquisition devices and postprocessing platforms to determine whether to administer thrombolytics. Simply put, it is questionable whether CT perfusion results generated by one protocol can be meaningfully compared with CT perfusion results derived using a different protocol.

The clinical significance of the widespread variability in CT perfusion results is difficult to estimate. Few studies have directly examined how CT perfusion affects clinical decision-making or influences patient outcomes, ${ }^{18}$ and the clinically acceptable limits of variability in CT perfusion results are unknown. These gaps in the medical literature call attention to the need for future studies to define the role of $\mathrm{CT}$ perfusion in acute stroke management. Future studies should take into consideration the widespread variability in CT perfusion results.

For quantitative CT perfusion results, standardization at all 3 stages of CT perfusion studies is warranted, and progress is being made. For example, in 2007, data acqui- 


\section{B. Zussman et al.}

sition (Stage 1) protocols were drafted at an international symposium of stroke imaging experts. ${ }^{28}$ Furthermore, the calibration of postprocessing software applications (Stage 2) against a digital, universal standard is increasingly likely, thanks to recent work of the ASIST-Japan group and the STIR consortium (Kudo et al., unpublished data, 2010). It is worth noting that although numerous factors cause variability in CT perfusion results, software design is a large factor, relative to other factors, ${ }^{32}$ which makes software standardization especially relevant. For qualitative CT perfusion results, standardized parametric-map representations of perfusion metrics are needed.

This study has several implications for current practice. First, caution should be exercised when interpreting CT perfusion results because they may vary considerably, depending upon a variety of factors. It remains unknown if, and to what extent, this variability may affect clinical decision-making or influence patient outcomes. Second, future studies evaluating CT perfusion should consider the finding that $\mathrm{CT}$ perfusion results generated by different techniques are not necessarily interchangeable. Third, standardization efforts at all 3 stages of the CT perfusion study cycle should be pursued.

\section{Disclosure}

The authors report no conflict of interest concerning the materials or methods used in this study or the findings specified in this paper.

Author contributions to the study and manuscript preparation include the following. Conception and design: Zussman. Acquisition of data: Zussman, Talekar. Analysis and interpretation of data: Zussman, Talekar, Flanders. Drafting the article: Zussman, Flanders. Critically revising the article: all authors. Approved the final version of the paper on behalf of all authors: Jabbour. Administrative/techni$\mathrm{cal} /$ material support: Talekar. Study supervision: Flanders.

\section{Acknowledgment}

The authors are grateful to Dr. K. Kudo, ASIST-Japan group, for kindly sharing the copies of 2 presentations on CT perfusion standardization.

\section{References}

1. Adams HP Jr, del Zoppo G, Alberts MJ, Bhatt DL, Brass L, Furlan A, et al: Guidelines for the early management of adults with ischemic stroke: A guideline from the American Heart Association/American Stroke Association Stroke Council, Clinical Cardiology Council, Cardiovascular Radiology and Intervention Council, and the Atherosclerotic Peripheral Vascular Disease and Quality of Care Outcomes in Research Interdisciplinary Working Groups: The American Academy of Neurology affirms the value of this guideline as an educational tool for neurologists. Circulation 115:e478-e534, 2007

2. Aviv RI, d'Esterre CD, Murphy BD, Hopyan JJ, Buck B, Mallia $\mathrm{G}$, et al: Hemorrhagic transformation of ischemic stroke: prediction with CT perfusion. Radiology 250:867-877, 2009

3. Bisdas S, Konstantinou GN, Gurung J, Lehnert T, Donnerstag F, Becker H, et al: Effect of the arterial input function on the measured perfusion values and infarct volumetric in acute cerebral ischemia evaluated by perfusion computed tomography. Invest Radiol 42:147-156, 2007

4. Ferreira RM, Lev MH, Goldmakher GV, Kamalian S, Schaefer PW, Furie KL, et al: Arterial input function placement for accurate CT perfusion map construction in acute stroke. AJR Am J Roentgenol 194:1330-1336, 2010
5. Fiorella D, Heiserman J, Prenger E, Partovi S: Assessment of the reproducibility of postprocessing dynamic CT perfusion data. AJNR Am J Neuroradiol 25:97-107, 2004

6. Hoeffner EG, Case I, Jain R, Gujar SK, Shah GV, Deveikis JP, et al: Cerebral perfusion CT: technique and clinical applications. Radiology 231:632-644, 2004

7. Hopyan J, Ciarallo A, Dowlatshahi D, Howard P, John V, Yeung $\mathrm{R}$, et al: Certainty of stroke diagnosis: incremental benefit with CT perfusion over noncontrast CT and CT angiography. Radiology 255:142-153, 2010

8. Kämena A, Streitparth F, Grieser C, Lehmkuhl L, Jamil B, Wojtal K, et al: Dynamic perfusion CT: optimizing the temporal resolution for the calculation of perfusion CT parameters in stroke patients. Eur J Radiol 64:111-118, 2007

9. Kealey SM, Loving VA, Delong DM, Eastwood JD: User-defined vascular input function curves: influence on mean perfusion parameter values and signal-to-noise ratio. Radiology 231:587-593, 2004

10. Kloska SP, Fischer T, Nabavi DG, Wessling J, Dittrich R, Fischbach R, et al: Comparison of different iodine concentration contrast media in perfusion computed tomography of the brain: is high iodine concentration useful? Invest Radiol 42:564-568, 2007

11. Kloska SP, Fischer T, Sauerland C, Buerke B, Dziewas R, Fischbach R, et al: Increasing sampling interval in cerebral perfusion CT: limitation for the maximum slope model. Acad Radiol 17:61-66, 2010

12. König M, Bültmann E, Bode-Schnurbus L, Koenen D, Mielke $\mathrm{E}$, Heuser L: Image quality in CT perfusion imaging of the brain. The role of iodine concentration. Eur Radiol 17:39-47, 2007

13. Kudo K, Sasaki M, Ogasawara K, Terae S, Ehara S, Shirato H: Difference in tracer delay-induced effect among deconvolution algorithms in CT perfusion analysis: quantitative evaluation with digital phantoms. Radiology 251:241-249, 2009

14. Kudo K, Sasaki M, Yamada K, Momoshima S, Utsunomiya H, Shirato H, et al: Differences in CT perfusion maps generated by different commercial software: quantitative analysis by using identical source data of acute stroke patients. Radiology 254:200-209, 2010

15. Kudo K, Terae S, Katoh C, Oka M, Shiga T, Tamaki N, et al: Quantitative cerebral blood flow measurement with dynamic perfusion CT using the vascular-pixel elimination method: comparison with $\mathrm{H} 2(15) \mathrm{O}$ positron emission tomography. AJNR Am J Neuroradiol 24:419-426, 2003

16. Latchaw RE, Yonas H, Hunter GJ, Yuh WT, Ueda T, Sorensen AG, et al: Guidelines and recommendations for perfusion imaging in cerebral ischemia: A scientific statement for healthcare professionals by the writing group on perfusion imaging, from the Council on Cardiovascular Radiology of the American Heart Association. Stroke 34:1084-1104, 2003

17. Murphy BD, Fox AJ, Lee DH, Sahlas DJ, Black SE, Hogan MJ, et al: White matter thresholds for ischemic penumbra and infarct core in patients with acute stroke: CT perfusion study. Radiology 247:818-825, 2008

18. Provenzale JM, Shah K, Patel U, McCrory DC: Systematic review of $\mathrm{CT}$ and MR perfusion imaging for assessment of acute cerebrovascular disease. AJNR Am J Neuroradiol 29: 1476-1482, 2008

19. Sanelli PC, Lev MH, Eastwood JD, Gonzalez RG, Lee TY: The effect of varying user-selected input parameters on quantitative values in CT perfusion maps. Acad Radiol 11:10851092, 2004

20. Sanelli PC, Nicola G, Johnson R, Tsiouris AJ, Ougorets I, Knight C, et al: Effect of training and experience on qualitative and quantitative CT perfusion data. AJNR Am J Neuroradiol 28:428-432, 2007

21. Sanelli PC, Nicola G, Tsiouris AJ, Ougorets I, Knight C, Frommer B, et al: Reproducibility of postprocessing of quantitative 


\section{Variability in computed tomography perfusion}

CT perfusion maps. AJR Am J Roentgenol 188:213-218, 2007

22. Sasaki M, Kudo K, Ogasawara K, Fujiwara S: Tracer delayinsensitive algorithm can improve reliability of CT perfusion imaging for cerebrovascular steno-occlusive disease: comparison with quantitative single-photon emission CT. AJNR Am J Neuroradiol 30:188-193, 2009

23. Soares BP, Dankbaar JW, Bredno J, Cheng S, Bhogal S, Dillon WP, et al: Automated versus manual post-processing of perfusion-CT data in patients with acute cerebral ischemia: influence on interobserver variability. Neuroradiology 51:445-451, 2009

24. Turk AS, Grayev A, Rowley HA, Field AS, Turski P, Pulfer $\mathrm{K}$, et al: Variability of clinical CT perfusion measurements in patients with carotid stenosis. Neuroradiology 49:955-961, 2007

25. van der Schaaf I, Vonken EJ, Waaijer A, Velthuis B, Quist M, van Osch T: Influence of partial volume on venous output and arterial input function. AJNR Am J Neuroradiol 27:46-50, 2006

26. Waaijer A, van der Schaaf IC, Velthuis BK, Quist M, van Osch MJ, Vonken EP, et al: Reproducibility of quantitative CT brain perfusion measurements in patients with symptomatic unilateral carotid artery stenosis. AJNR Am J Neuroradiol 28:927-932, 2007

27. Wiesmann M, Berg S, Bohner G, Klingebiel R, Schöpf V, Stoeckelhuber BM, et al: Dose reduction in dynamic perfusion CT of the brain: effects of the scan frequency on measurements of cerebral blood flow, cerebral blood volume, and mean transit time. Eur Radiol 18:2967-2974, 2008
28. Wintermark M, Albers GW, Alexandrov AV, Alger JR, Bammer R, Baron JC, et al: Acute stroke imaging research roadmap. AJNR Am J Neuroradiol 29:e23-e30, 2008

29. Wintermark M, Fischbein NJ, Smith WS, Ko NU, Quist M, Dillon WP: Accuracy of dynamic perfusion CT with deconvolution in detecting acute hemispheric stroke. AJNR Am J Neuroradiol 26:104-112, 2005

30. Wintermark M, Lau BC, Chien J, Arora S: The anterior cerebral artery is an appropriate arterial input function for perfusion-CT processing in patients with acute stroke. Neuroradiology 50:227-236, 2008

31. Wintermark M, Smith WS, Ko NU, Quist M, Schnyder P, Dillon WP: Dynamic perfusion CT: optimizing the temporal resolution and contrast volume for calculation of perfusion CT parameters in stroke patients. AJNR Am J Neuroradiol 25:720-729, 2004

32. Zussman B, Boghosian G, Gorniak R, Olszewski M, Read K, Siddiqui K, et al: The relative effect of vendor variability in computed tomography perfusion (CTP) results: a methodcomparison study. AJR Am J Roentgenol [in press], 2011

Manuscript submitted February 9, 2011.

Accepted March 7, 2011.

Address correspondence to: Pascal Jabbour, M.D., Department of Neurosurgery, Division of Neurovascular Surgery and Endovascular Neurosurgery, Thomas Jefferson University Hospital, 909 Walnut Street, 2nd Floor, Philadelphia, Pennsylvania 19107. email: pascal. jabbour@jefferson.edu. 